\title{
Research Article \\ Regge Poles in Neutron Scattering by a Cylinder
}

\author{
K. V. Protasov' ${ }^{1}$ and A. Y. Voronin ${ }^{2}$ \\ ${ }^{1}$ Laboratoire de Physique Subatomique et de Cosmologie (LPSC), Université Grenoble Alpes, IN2P3-CNRS, \\ 53 Avenue des Martyrs, 38026 Grenoble, France \\ ${ }^{2}$ P.N. Lebedev Physical Institute, 53 Leninsky Prospekt, Moscow 119991, Russia
}

Correspondence should be addressed to A. Yu. Voronin; dr.a.voronin@gmail.com

Received 20 July 2014; Accepted 24 August 2014; Published 16 September 2014

Academic Editor: Valery V. Nesvizhevsky

Copyright (C) 2014 K.V. Protasov and A. Yu. Voronin. This is an open access article distributed under the Creative Commons Attribution License, which permits unrestricted use, distribution, and reproduction in any medium, provided the original work is properly cited. The publication of this article was funded by SCOAP ${ }^{3}$.

We establish asymptotic expressions for the positions of Regge poles for cold neutron scattering on mesoscopic cylinder mirror as well as for the $S$-matrix residuals. We outline the correspondence between Regge poles and near-surface quasi-stationary neutron states. Such states are of practical importance for studying subtle effects of neutron-surface interaction.

\section{Introduction}

Neutron scattering on curved surface has all peculiar features characteristic for wave scattering known in acoustics, optics, and matter waves. The most intriguing phenomena are related to localization of neutrons in long-living states near a curved surface. Such localization is responsible for large angle scattering of cold neutrons on macroscopic radius cylinder mirror, discovered in [1-4]. It is a matter-wave analog of the so-called whispering gallery wave [5-10].

Neutron localization in a resonant state in effective potential, which originates from superposition of centrifugal potential and optical Fermi-potential of the cylinder, results in a large time spent by neutron near the material surface at distances from tens to hundreds nanometers. Thus neutron scattering on curved mirror is particularly sensitive to neutron-surface interaction at such distances. This opens interesting perspective of using interference pattern produced by neutrons scattered from curved surface as a sensitive tool for studying neutron-surface interactions. The detailed information about neutron-surface interaction could be of importance for multiple problems related to surface physics. Another important field is the study of extra forces with characteristic range $10<\lambda<1000 \mathrm{~nm}$. These forces are predicted in the extensions of the standard model as a result of exchange of hypothetical light bosons.
We have developed an approach based on a linear approximation of the potential in Schrödinger equation in [4]. Here we propose an alternative method based on exact solution with consequent approximations in the exact result. The approach obtained in present paper could be applied at least formally in wider parameter range than that obtained in [4] and, in particular, for neutrons of lower energies. This method allows us also to give another vision to the problem and to establish more clear analogies with another extensively studied case, that is, scattering of light by a sphere and, in particular, useful similarities with phenomena of rainbow, Gloria, surface waves, and so forth, treated in detail in a book by Nussenzweig [11]. The complex angular momentum (CAM) method plays an essential role in this study.

The concept of Regge poles, which are $S$-matrix singularities, thought of as a function of complex angular momentum is essential for establishing relation between scattering phenomena and the properties of long-living nearsurface states of neutron.

In this paper we find asymptotic expressions for the positions of Regge poles in the limit of large angular momenta, which mainly contribute to large angle scattering of cold neutrons. We study a case of cold neutron scattering, as far as comparatively large amount of cold neutrons available makes possible high statistical accuracy in resolving interference pattern of scattered neutron wave. 


\section{Formal Solution and Regge Poles}

In this section we develop the formalism describing the scattering of a plane neutron wave by a cylindrical mirror. We will study cases of both attractive and repulsive optical Fermi-potential of a cylinder material.

The scattering obeys the following Schrödinger equation in the cylindrical coordinates:

$$
\begin{aligned}
& {\left[-\frac{\hbar^{2}}{2 M}\left(\frac{\partial^{2}}{\partial \rho^{2}}+\frac{1}{\rho} \frac{\partial}{\partial \rho}\right)-\frac{\hbar^{2}}{2 M \rho^{2}} \frac{\partial^{2}}{\partial \varphi^{2}}+U(\rho)-\frac{\hbar^{2} k^{2}}{2 M}\right]} \\
& \times \Psi(\rho, \varphi)=0 .
\end{aligned}
$$

Here $\Psi(\rho, \varphi)$ is the neutron wave function, $\hbar k$ is the neutron momentum, $\rho$ is the radial distance from the cylinder axis, $\varphi$ is the angle, and $U(\rho)$ is the value of mirror optical potential:

$$
U(\rho)=U_{0} \Theta(R-\rho)= \begin{cases}U_{0}, & \rho \leq R \\ 0, & \rho>R\end{cases}
$$

Here $R$ is the cylinder radius and $\Theta(R-\rho)$ is the step function. $U_{0}$ is negative for attractive potential and positive for repulsion one. In (1), we omit the trivial dependence on $z$ coordinate directed along the cylinder axis. By standard substitution of an analytical form of a wave function $\Psi(\rho, \varphi)=$ $\Phi(\rho, \varphi) / \sqrt{\rho}$, (1) is transformed into the following form:

$$
\begin{aligned}
& {\left[-\frac{\hbar^{2}}{2 M}\left(\frac{\partial^{2}}{\partial \rho^{2}}\right)-\frac{\hbar^{2}}{2 M \rho^{2}}\left(\frac{\partial^{2}}{\partial \varphi^{2}}+\frac{1}{4}\right)+U_{0} \Theta(R-\rho)-\frac{\hbar^{2} k^{2}}{2 M}\right]} \\
& \quad \times \Phi(\rho, \varphi)=0 .
\end{aligned}
$$

The wave function $\Phi(\rho, \varphi)$ asymptotic behavior at large $\rho$ values is

$$
\Phi(\rho, \varphi) \longrightarrow \sqrt{\rho} \exp (i k \rho \cos (\varphi))+f(\varphi) \exp \left(i k \rho+\frac{i \pi}{4}\right),
$$

where $f(\varphi)$ is the scattering amplitude. The standard expansion of the two-dimensional wave function $\Phi(\rho, \varphi)$ in the complete basis of the angular momentum states $\exp (i \mu \varphi)$ is

$$
\Phi(\rho, \varphi)=\sum_{\mu=-\infty}^{\infty} \chi_{|\mu|}(\rho) e^{i \mu \varphi}
$$

where $\chi_{|\mu|}(\rho)$ are the radial wave functions.

The scattering amplitude $f(\varphi)$ expansion in the complete basis of the angular momentum states $\exp (i \mu \varphi)$ follows from (5) and has the following form [12]:

$$
f(\varphi)=\frac{-i}{\sqrt{2 \pi k}} \sum_{\mu=-\infty}^{\infty}(S(\mu, k)-1) e^{i \mu \varphi} \equiv \sum_{\mu=-\infty}^{\infty} f(\mu, k) e^{i \mu \varphi} .
$$

Here $S(\mu, k)=\exp \left(2 i \delta_{\mu}(k)\right)$ is the scattering matrix in partial wave with angular momentum $\mu$ and $\delta_{\mu}(k)$ is the scattering phase shift, which can be found by solving the corresponding radial equation:

$$
\begin{aligned}
& {\left[-\frac{\hbar^{2}}{2 M}\left(\frac{\partial^{2}}{\partial \rho^{2}}\right)+\frac{\hbar^{2}}{2 M \rho^{2}}\left(\mu^{2}-\frac{1}{4}\right)+U_{0} \Theta(R-\rho)-\frac{\hbar^{2} k^{2}}{2 M}\right]} \\
& \times \chi_{\mu}(\rho)=0,
\end{aligned}
$$

$$
\begin{gathered}
\chi_{\mu}(\rho \longrightarrow 0)=0 \\
\chi_{\mu}(\rho \longrightarrow \infty)=\sqrt{\frac{2}{\pi k}} \sin \left(k \rho+\delta_{\mu}-\frac{\pi}{2}\left(|\mu|-\frac{1}{2}\right)\right) .
\end{gathered}
$$

The typical values of angular momentum $\mu_{0} \equiv k R \gg 1$. In particular, in case of cold neutron scattering on macroscopic cylinder with few centimeters radius the corresponding value $\mu_{0} \sim 10^{8}$.

In order to perform a sum over a large number of partial waves we will use the method of complex angular momentum. We introduce, following the Regge approach, $f(\mu, k)$ as a function of complex momentum $\mu$, which coincides with the scattering matrix for integer values of $\mu$ and has standard analytical properties in the complex plane of $\mu[13,14]$. The sum (6) over integer $\mu$ is then transformed to an integral in the complex $\mu$ plane, which is calculated by using the residue theorem and is replaced by a sum over poles contributions. In two-dimensional problems, an elegant way to perform such a transformation is to use Poisson sum formula [15]:

$$
f(\varphi)=\sum_{n=-\infty}^{\infty} \int_{-\infty}^{\infty} f(\mu, k) e^{i \mu(\varphi+2 \pi n)} d \mu .
$$

Here $f(\mu, k)$ is the analytical function of $\mu$ as mentioned above. The integer number $n$ has a sense of the number of neutron wave rotations around the cylinder surface. Using the analytical properties of the amplitude we transform the above integral to the sum of the amplitude poles contribution:

$$
f(\varphi)=2 \pi i \sum_{n=-\infty}^{\infty} \sum_{j} \operatorname{Res} f\left(\mu_{j}, k\right) e^{i \mu_{j}(\varphi+2 \pi n)} .
$$

Here $\mu_{j}$ is the $j$ th pole of the amplitude $f$ and $\operatorname{Res} f\left(\mu_{j}, k\right)$ is a residue of the amplitude in this pole. In the above expression the integration contour is chosen such that $\operatorname{Im} \mu_{j} \varphi>0$ and $\operatorname{Im} \mu_{j}(\pi-\varphi)>0$. The summation over $n$ can be performed after taking into account that the amplitude in our case is symmetric under substitution $\mu \rightarrow-\mu$. Such an expression takes the form

$$
f(\varphi)=2 \pi i \sum_{j} \operatorname{Res} f\left(\mu_{j}, k\right) \frac{\sin \left(\mu_{j}(\pi-\varphi)\right)}{\sin \left(\mu_{j} \pi\right)} .
$$

The above expression clearly demonstrates the role of Regge poles; namely, the scattering amplitude can be expressed as a converging sum of Regge poles contributions. Each contribution can be associated with decaying quasistationary states. The states with the longest lifetime contribute to scattering on largest deflection angles. In the following section we will get formal mathematical expressions for the Regge poles and discuss their physical meaning. 
2.1. Equation for Regge Poles. The formal solution of (3) can be easily expressed in terms of Bessel functions.

Inside the cylinder $\rho<R$, the regular solution (8) of (7) is proportional to the Bessel function

$$
\chi_{\mu}(\rho) \sim J_{\mu}(\kappa \rho)
$$

with $\kappa=\sqrt{k^{2}-2 M U_{0} / \hbar^{2}} \approx k-M U_{0} / k \hbar^{2}$. Let us remind the reader that $U_{0}$ is negative for attractive potential and positive for repulsion one. Outside the cylinder one has a sum of two independent solutions of Bessel equation:

$$
\chi_{\mu}(\rho) \sim \cos \delta_{\mu} J_{\mu}(k \rho)+\sin \delta_{\mu} N_{\mu}(k \rho) .
$$

The condition of continuity of the logarithmic derivative of the wave function on the boundary of the cylinder $\rho=R$ gives the equation

$$
\kappa \frac{J_{\mu}^{\prime}(\kappa R)}{J_{\mu}(\kappa R)}=k \frac{\cos \delta_{\mu} J_{\mu}^{\prime}(k R)+\sin \delta_{\mu} N_{\mu}^{\prime}(k R)}{\cos \delta_{\mu} J_{\mu}(k R)+\sin \delta_{\mu} N_{\mu}(k R)}
$$

which allows us to determine the value of the phase-shift $\delta_{\mu}$.

Finally, one obtains

$$
\tan \delta_{\mu}=\frac{(\kappa / k)\left(J_{\mu}^{\prime}(\kappa R) / J_{\mu}(\kappa R)\right) J_{\mu}(k R)-J_{\mu}^{\prime}(k R)}{(\kappa / k)\left(J_{\mu}^{\prime}(\kappa R) / N_{\mu}(\kappa R)\right) J_{\mu}(k R)-N_{\mu}^{\prime}(k R)} .
$$

For the $S$-matrix, one obtains

$$
\begin{aligned}
S(\mu, p)= & \exp \left(2 i \delta_{\mu}\right), \\
S(\mu, p)= & \left(N_{\mu}^{\prime}(k R)+i J_{\mu}^{\prime}(k R)\right. \\
& \left.\quad-\frac{\kappa}{k} \frac{J_{\mu}^{\prime}(\kappa R)}{J_{\mu}(\kappa R)}\left(N_{\mu}(k R)+i J_{\mu}(k R)\right)\right) \\
& \times\left(N_{\mu}^{\prime}(k R)-i J_{\mu}^{\prime}(k R)\right. \\
& \left.\quad-\frac{\kappa}{k} \frac{J_{\mu}^{\prime}(\kappa R)}{J_{\mu}(\kappa R)}\left(N_{\mu}(k R)-i J_{\mu}(k R)\right)\right)^{-1}
\end{aligned}
$$

which coincides with the solution for 3-dimensional problem (scattering by a spherical potential well) if one replaces $\mu \rightarrow$ $l+1 / 2$.

These general expressions for Bessel $J_{\mu}(z)$ and Neumann $N_{\mu}(z)$ functions can be simplified in the limit of very big index $\mu$ which is close to the value of the argument of the Bessel function:

$$
\mu \sim \mu_{0} \equiv k R \sim \kappa R \gg 1 .
$$

Let us use the asymptotic for the Bessel function [16] in the form $J_{\mu}(\mu z)$ with two complex variables $\mu$ and $z$.
In the limit of large index one can use the following asymptotic behaviour for Bessel functions (only a leading in $\mu$ term is written here):

$$
J_{\mu}(\mu z) \approx\left(\frac{4 \zeta}{1-z^{2}}\right)^{1 / 4} \frac{\mathrm{Ai}\left(\mu^{2 / 3} \zeta\right)}{\mu^{1 / 3}},
$$

where

$$
\frac{2}{3} \zeta^{3 / 2}=\int_{z}^{1} \frac{\sqrt{1-t^{2}}}{t} d t=\ln \frac{1+\sqrt{1-z^{2}}}{z}-\sqrt{1-z^{2}}
$$

or equivalently

$$
\frac{2}{3}(-\zeta)^{3 / 2}=\int_{1}^{z} \frac{\sqrt{t^{2}-1}}{t} d t=\sqrt{z^{2}-1}-\frac{1}{i} \ln \frac{1+i \sqrt{z^{2}-1}}{z} .
$$

$\operatorname{Ai}(z)$ is the Airy function.

For $z$ close to 1 ,

$$
\zeta \approx 2^{1 / 3}(z-1)
$$

Analogous expression can be obtained for Neumann function as well as for the derivatives of Bessel and Neumann functions

$$
\begin{aligned}
& N_{\mu}(\mu z) \approx-\left(\frac{4 \zeta}{1-z^{2}}\right)^{1 / 4} \frac{\mathrm{Bi}\left(\mu^{2 / 3} \zeta\right)}{\mu^{1 / 3}}, \\
& J_{\mu}^{\prime}(\mu z) \approx-\frac{2}{z}\left(\frac{1-z^{2}}{4 \zeta}\right)^{1 / 4} \frac{\mathrm{Ai}^{\prime}\left(\mu^{2 / 3} \zeta\right)}{\mu^{2 / 3}}, \\
& N_{\mu}^{\prime}(\mu z) \approx \frac{2}{z}\left(\frac{1-z^{2}}{4 \zeta}\right)^{1 / 4} \frac{\mathrm{Bi}^{\prime}\left(\mu^{2 / 3} \zeta\right)}{\mu^{2 / 3}} .
\end{aligned}
$$

$\operatorname{Bi}(z)$ is an Airy function of the second kind.

If one introduces the notations

$$
\zeta_{\text {in }}=2^{1 / 3}\left(\frac{\kappa R}{\mu}-1\right), \quad \zeta_{\text {out }}=2^{1 / 3}\left(\frac{k R}{\mu}-1\right),
$$

one finds the expression for the $S$-matrix

$$
S(\mu, k)=\frac{T^{-}(\mu, k)}{T^{+}(\mu, k)}
$$

with

$$
\begin{aligned}
T^{ \pm}(\mu, k)= & \operatorname{Bi}^{\prime}\left(\mu^{2 / 3} \zeta_{\text {out }}\right) \pm i \operatorname{Ai}^{\prime}\left(\mu^{2 / 3} \zeta_{\text {out }}\right) \\
& -\frac{\operatorname{Ai}^{\prime}\left(\mu^{2 / 3} \zeta_{\text {in }}\right)}{\operatorname{Ai}\left(\mu^{2 / 3} \zeta_{\text {in }}\right)}\left[\operatorname{Bi}\left(\mu^{2 / 3} \zeta_{\text {out }}\right) \pm i \operatorname{Ai}\left(\mu^{2 / 3} \zeta_{\text {out }}\right)\right] .
\end{aligned}
$$

The poles of the $S$-matrix can be found from the equation

$$
T^{+}(\mu, k)=0,
$$

or in explicit form:

$$
\frac{\operatorname{Ai}^{\prime}\left(\mu^{2 / 3} \zeta_{\text {in }}\right)}{\operatorname{Ai}\left(\mu^{2 / 3} \zeta_{\text {in }}\right)}=\frac{\operatorname{Bi}^{\prime}\left(\mu^{2 / 3} \zeta_{\text {out }}\right)+i \mathrm{Ai}^{\prime}\left(\mu^{2 / 3} \zeta_{\text {out }}\right)}{\operatorname{Bi}\left(\mu^{2 / 3} \zeta_{\text {out }}\right)+i \operatorname{Ai}\left(\mu^{2 / 3} \zeta_{\text {out }}\right)} .
$$


2.2. Asymptotic Expressions for Regge Poles. In the following we will be interested in the extremely large values of angular momentum. These are angular momenta which contribute to large angle neutron scattering. Indeed, the energy of cold neutrons is much larger than optical Fermi-potential of a cylinder; thus, most neutrons would pass without significant deflection. Only a small fraction of neutrons which moves parallel to the cylinder surface is captured into comparatively long-living near-surface states, which explains large angle scattering.

An argument of Airy function equation (31) can be further expanded, taking into account large values of $\mu \sim$ $\mu_{0} \gg 1$ :

$$
\begin{aligned}
\mu^{2 / 3} \zeta_{\text {in }} & =\left(\frac{2}{\mu}\right)^{1 / 3}(\kappa R-\mu) \approx-u_{0}-x m, \\
\mu^{2 / 3} \zeta_{\text {out }} & =\left(\frac{2}{\mu}\right)^{1 / 3}(k R-\mu)=-x .
\end{aligned}
$$

Here,

$$
\begin{gathered}
u_{0}=\frac{M U_{0} R}{\hbar^{2} k}\left(\frac{2}{\mu_{0}}\right)^{1 / 3}=\frac{2 M U_{0}}{\hbar^{2} k^{2}}\left(\frac{\mu_{0}}{2}\right)^{2 / 3}, \\
x=\left(\mu-\mu_{0}\right)\left(\frac{2}{\mu_{0}}\right)^{1 / 3} .
\end{gathered}
$$

The complex variable $x$ is proportional to the difference between actual angular momentum $\mu$ and "edge scattering" angular momentum $\mu_{0}$.

Equation (31) for the Regge poles in new variable $x_{n}$ takes the form

$$
\frac{\operatorname{Ai}^{\prime}\left(-u_{0}-x_{n}\right)}{\operatorname{Ai}\left(-u_{0}-x_{n}\right)}=\frac{\operatorname{Bi}^{\prime}\left(-x_{n}\right)+i \mathrm{Ai}^{\prime}\left(-x_{n}\right)}{\operatorname{Bi}\left(-x_{n}\right)+i \operatorname{Ai}\left(-x_{n}\right)} .
$$

This form of equation is convenient for further asymptotic expansions for large values of $x_{n}$. The particular form of asymptotic expansion of Airy function in complex plane of $x$ depends on the argument of $x$. This is known as Stock's phenomenon. Thus we will study different domains of complex momentum plane.

First we study a case of narrow resonances which are situated close to real axis of complex momentum plane. In this case the following asymptotic form of Airy functions can be used:

$$
\begin{aligned}
& \operatorname{Ai}(-z) \sim \frac{1}{\sqrt{\pi} z^{1 / 4}} \cos \left(\frac{2}{3} z^{3 / 2}-\frac{\pi}{4}\right), \\
& \operatorname{Bi}(-z) \sim-\frac{1}{\sqrt{\pi} z^{1 / 4}} \sin \left(\frac{2}{3} z^{3 / 2}-\frac{\pi}{4}\right)
\end{aligned}
$$

with $|\arg (z)|<2 \pi / 3$ and

$$
\begin{aligned}
& \operatorname{Ai}(z) \sim \frac{1}{2 \sqrt{\pi} z^{1 / 4}} \exp \left(-\frac{2}{3} z^{3 / 2}\right) \quad \text { with }|\arg (z)|<\pi, \\
& \operatorname{Bi}(z) \sim \frac{1}{\sqrt{\pi} z^{1 / 4}} \exp \left(\frac{2}{3} z^{3 / 2}\right) \quad \text { with }|\arg (z)|<\frac{\pi}{3} .
\end{aligned}
$$

In case of attractive potential $\left(u_{0}<0\right)$ there could be poles with $u_{0}<\operatorname{Re} x_{n}<0,\left|\operatorname{Im} x_{n}\right| \ll\left|\operatorname{Re} x_{n}\right|$. For $\left|u_{0}\right| \gg 1$ (34) takes the form

$$
\begin{gathered}
\sqrt{\frac{\left|u_{0}\right|+x_{n}}{-x_{n}}} \tan \left[\frac{2}{3}\left(\left|u_{0}\right|+x_{n}\right)^{3 / 2}-\frac{\pi}{4}\right] \\
=1-i \exp \left[-\frac{4}{3}\left(-x_{n}\right)^{3 / 2}\right] .
\end{gathered}
$$

The approximate solution of the above equation gives exponentially small values for the imaginary part:

$$
\operatorname{Im} x_{n} \sim \exp \left[-\frac{4}{3} \operatorname{Re}\left(-x_{n}\right)^{3 / 2}\right],
$$

with real part $\operatorname{Re} x_{n}$ being a solution of equation:

$$
\sqrt{\frac{\left|u_{0}\right|+x_{n}}{-x_{n}}} \tan \left[\frac{2}{3}\left(\left|u_{0}\right|+x_{n}\right)^{3 / 2}-\frac{\pi}{4}\right]=1 .
$$

In the limiting case of deeply bound states ||$u_{0}\left|+x_{n}\right| \ll$ $\left|x_{n}\right|$ one gets, for real part $\operatorname{Re} x_{n}$,

$$
\operatorname{Re} x_{n} \simeq-\left[\frac{3}{2} \pi\left(n-\frac{1}{4}\right)\right]^{2 / 3} .
$$

In case of weakly bound states ||$u_{0}\left|+x_{n}\right| \gg\left|x_{n}\right|$,

$$
\operatorname{Re} x_{n} \simeq-\left[\frac{3}{2} \pi\left(n-\frac{3}{4}\right)\right]^{2 / 3} \text {. }
$$

Here and further on $n=1,2, \ldots$.

There are a limited number of such resonances determined by the value of dimensionless depth of optical potential $u_{0}$.

Narrow resonances $\left|\operatorname{Im} x_{n}\right| \ll\left|\operatorname{Re} x_{n}\right|$ with positive real part $\operatorname{Re} x_{n}>0$ in case of attractive potential and $\operatorname{Re} x_{n}>$ $u_{0}$ in case of repulsive potential are given by the following asymptotic form of (34):

$$
\tan \left[\frac{2}{3}\left(x_{n}-u_{0}\right)^{3 / 2}-\frac{\pi}{4}\right]=i \sqrt{\frac{x_{n}}{x_{n}-u_{0}}}
$$

Its approximative solutions are given by the following expressions:

$$
\begin{gathered}
\operatorname{Re} x_{n}=\left[\frac{3}{2} \pi\left(n-\frac{3}{4}\right)\right]^{2 / 3}+\left|u_{0}\right| \Theta\left(U_{0}\right), \\
\operatorname{Im} x_{n}=-\frac{1}{\sqrt{\operatorname{Re} x_{n}}}\left(\frac{1}{2} \ln \frac{4 \operatorname{Re} x_{n}}{\left|u_{0}\right|}-\frac{\left|u_{0}\right|}{4 \operatorname{Re} x_{n}}\right) .
\end{gathered}
$$

Here $\Theta\left(U_{0}\right)$ is equal to unity for repulsive potential and is equal to zero for attractive potential.

Formally, there are an infinite number of mentioned above-barrier resonances. However, for too large $n$ the approximation based on asymptotic expansion of Bessel functions (20) and (24) is no longer valid, as far as their validity is limited with cases when $\left|\mu-\mu_{0}\right| \ll \mu_{0}$. In opposite 
case one has to take into account exact form of the $S$-matrix, given by (18).

These are the Regge poles of so-called class I [14] corresponding to the narrow resonances. The corresponding values of complex angular momentum $\mu_{n}$ are

$$
\mu_{n}= \pm\left[\mu_{0}-x_{n}\left(\frac{\mu_{0}}{2}\right)^{1 / 3}\right] \text {. }
$$

They are situated in the complex $\mu$-plane symmetrically related to the origin $\mu=0$.

Long-living quasi-bound states and narrow over-barrier resonances play important role in the phenomena of whispering gallery scattering of neutrons at large angles, studied in [4].

Another type of asymptotic expansion is obtained when the argument of the Airy functions $|\arg (z)| \geq 2 \pi / 3$. To study this case we make the following substitution:

$$
x_{n}=-z_{n} \exp \left(\frac{i \pi}{3}\right) \text {. }
$$

With the use of the following relations between Airy functions

$$
\begin{aligned}
& \operatorname{Ai}(-z)=e^{i \pi / 3} \operatorname{Ai}\left(z e^{i \pi / 3}\right)+e^{-i \pi / 3} \operatorname{Ai}\left(z e^{-i \pi / 3}\right), \\
& \operatorname{Bi}(-z)=e^{-i \pi / 6} \operatorname{Ai}\left(z e^{i \pi / 3}\right)+e^{i \pi / 6} \operatorname{Ai}\left(z e^{-i \pi / 3}\right),
\end{aligned}
$$

(34) turns into

$$
\frac{\operatorname{Ai}^{\prime}\left(u_{0}+z_{n} e^{i \pi / 3}\right)}{\operatorname{Ai}\left(u_{0}+z_{n} e^{i \pi / 3}\right)}=\frac{\operatorname{Ai}^{\prime}\left(-z_{n}\right)}{\operatorname{Ai}\left(-z_{n}\right)} .
$$

For $\left|z_{n}\right| \gg 1$ we get the following asymptotic form equation:

$$
\tan \left(\frac{2}{3} z_{n}^{3 / 2}-\frac{\pi}{4}\right)=\sqrt{\frac{z_{n} e^{i \pi / 3}+u_{0}}{z_{n}}} .
$$

Its solution for large $n$ is

$$
z_{n}=\left\{\frac{3 \pi}{2}\left[n-\frac{3}{4}+\arctan \left(e^{i \pi / 6}\right)\right]\right\}^{2 / 3} .
$$

The corresponding Regge poles are

$$
\mu_{n}= \pm\left(\mu_{0}+z_{n} e^{i \pi / 3}\left(\frac{\mu_{0}}{2}\right)^{1 / 3}\right) .
$$

There are an infinite number of Regge poles of this type; their imaginary part increases rapidly with $n$ and they correspond to the so-called surface wave states. Such states have much shorter lifetimes than narrow resonances, described above.

The positions of these poles in the upper half plane of complex variable $\mu$ are presented in Figures 1 and 2 for attractive and repulsive potential, respectively.

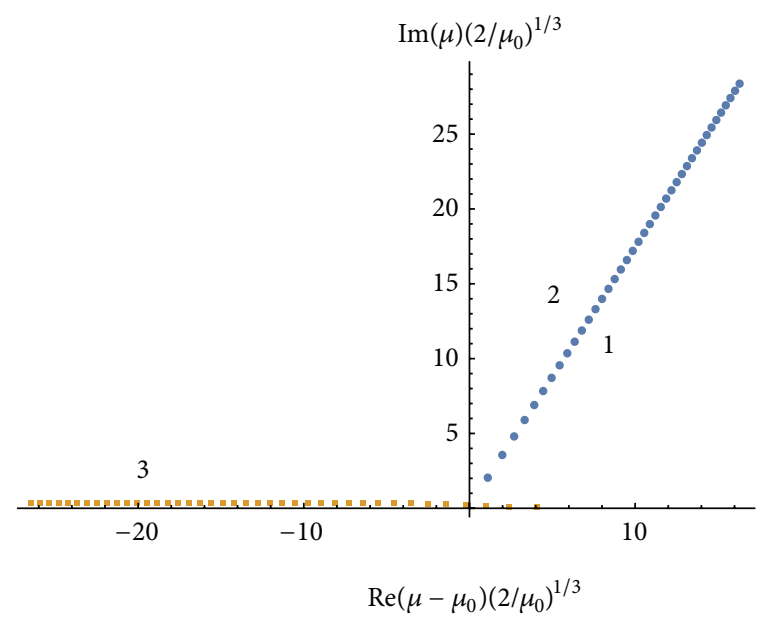

FIgURE 1: Position of Regge poles for attractive Fermi-potential. Shown are the poles, corresponding to near-surface states. 1: narrow quasi-bound states (whispering gallery states), 2: surface waves, and 3: narrow over-barrier resonances.

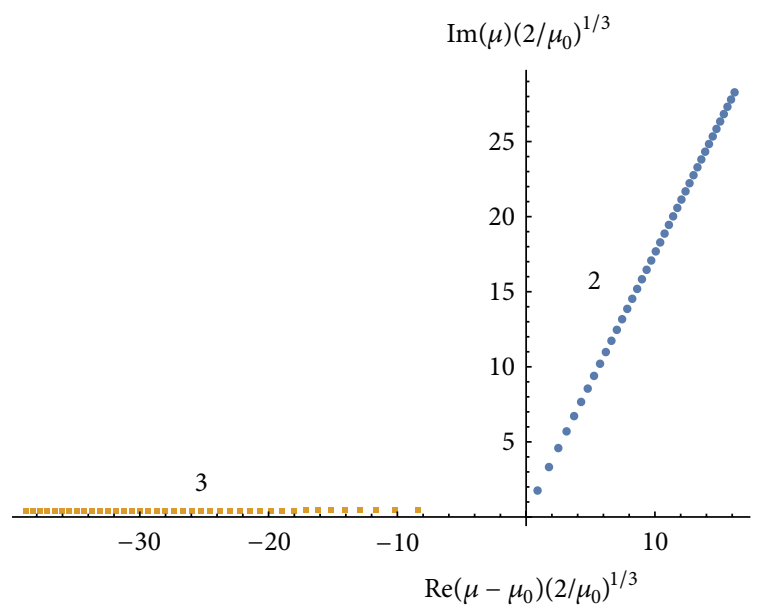

FIgURE 2: Position of Regge poles for repulsive Fermi-potential. Shown are the poles, corresponding to near-surface states. 2: surface waves and 3: narrow over-barrier resonances.

\section{Residuals}

Complex angular momentum methods require knowledge of the residuals of $S$-matrix in the Regge poles. Here we obtain corresponding expressions for residuals, which follow from expression (28). In the vicinity of the pole $x_{n}$ the corresponding $S$-matrix written in variable $x$ is

$$
S(x) \approx \frac{T^{-}\left(x_{n}\right)}{T^{+^{\prime}}\left(x_{n}\right)\left(x-x_{n}\right)} .
$$

Function $T$ in the above expression has the form

$$
\begin{aligned}
T^{ \pm}(x)= & \operatorname{Ai}\left(-u_{0}-x\right)\left(\mathrm{Bi}^{\prime}(-x) \pm i \mathrm{Ai}^{\prime}\right) \\
& -\mathrm{Ai}^{\prime}\left(-u_{0}-x\right)(\operatorname{Bi}(-x) \pm i \operatorname{Ai}(-x)) .
\end{aligned}
$$


Thus residual is

$$
\operatorname{Res} S\left(x_{n}\right)=\frac{T^{-}\left(x_{n}\right)}{T^{+^{\prime}}\left(x_{n}\right)}
$$

The above expression can be further simplified if one takes into account that $x_{n}$ is a root of equation $T^{+}\left(x_{n}\right)=0$. One can get for the nominator of fraction (53) the following expression:

$$
T^{-}\left(x_{n}\right)=-\frac{2 i}{\pi} \frac{\mathrm{Ai}\left(-u_{0}-x_{n}\right)}{\operatorname{Bi}\left(-x_{n}\right)+i \operatorname{Ai}\left(-x_{n}\right)} .
$$

In derivation of the above equation we took into account that the Wronskian $\mathrm{Ai}^{\prime}(-x) \mathrm{Bi}(-x)-\mathrm{Bi}^{\prime}(-x) \mathrm{Ai}(-x)=1 / \pi$.

The corresponding expression for the denominator of fraction (53) reads

$$
T^{+^{\prime}}\left(x_{n}\right)=-u_{0} \operatorname{Ai}\left(-u_{0}-x_{n}\right)\left(\operatorname{Bi}\left(-x_{n}\right)+i \operatorname{Ai}\left(-x_{n}\right)\right) .
$$

One should use the equation for Airy functions $\mathrm{Ai}^{\prime \prime}(-x)=$ $x \operatorname{Ai}(-x)$ to establish the above expression.

Combining these results we finally get the following expression for the residual:

$$
\operatorname{Res} S\left(x_{n}\right)=\frac{2 i}{\pi u_{0}\left(\operatorname{Bi}\left(-x_{n}\right)+i \operatorname{Ai}\left(-x_{n}\right)\right)^{2}} .
$$

\section{Physical Meaning of Regge Poles}

In order to establish physical meaning of the Regge poles found in the previous section we will establish a relation between Regge poles and quasi-stationary states of neutrons near a curved surface of macroscopic mirror. For such a purpose we will study an effective potential produced by superposition of optical Fermi-potential of the mirror and the centrifugal potential near the mirror surface.

We expand the expression for the centrifugal energy in (7) in the vicinity of $\rho=R$ introducing the deviation from the cylinder surface $\xi=\rho-R$. In the first order of small ratio $\xi / R$, we get the following equation:

$$
\begin{aligned}
& {\left[-\frac{\hbar^{2}}{2 M} \frac{\partial^{2}}{\partial \xi^{2}}+U_{0} \Theta(-\xi)+\hbar^{2} \frac{\mu^{2}-1 / 4}{2 M R^{2}}\left(1-\frac{2 \xi}{R}\right)-E\right]} \\
& \times \chi_{\mu}(\xi)=0 .
\end{aligned}
$$

Introducing a new variable

$$
\varepsilon_{\mu}=E-\hbar^{2} \frac{\mu^{2}-1 / 4}{2 M R^{2}} \simeq \frac{(M v R)^{2}-\hbar^{2} \mu^{2}}{2 M R^{2}}
$$

we get the following equation for the neutron radial motion near the surface of the cylinder:

$$
\left[-\frac{\hbar^{2}}{2 M} \frac{\partial^{2}}{\partial \xi^{2}}+U_{0} \Theta(-\xi)-\frac{M v^{2}}{R} \xi-\varepsilon_{\mu}\right] \chi_{\mu}(\xi)=0 .
$$

Equation (59) describes the neutron motion in a constant effective field $a=-v^{2} / R$ superposed with the mirror optical potential $U_{0} \Theta(-\xi)$. A sketch of the corresponding potential for attractive and repulsive optical potential is shown in Figure 3 . The value $\varepsilon_{\mu}$ can be understood as the radial motion energy within the linear expansion used above for the angular momentum $\left|\mu-\mu_{0}\right| \ll \mu_{0}$.

Characteristic spatial and energy scales of the above equation are

$$
\begin{gathered}
l_{0}=\sqrt[3]{\frac{\hbar^{2} R}{2 M^{2} v^{2}}}=\frac{R}{2}\left(\frac{2}{\mu_{0}}\right)^{2 / 3}, \\
\varepsilon_{0}=\sqrt[3]{\frac{\hbar^{2} M v^{4}}{2 R^{2}}}=\frac{M v^{2}}{2}\left(\frac{2}{\mu_{0}}\right)^{2 / 3} .
\end{gathered}
$$

Equation (59) in units of $l_{0}$ and $\varepsilon_{0}$ is

$$
\left[-\frac{\partial^{2}}{\partial x^{2}}+u_{0} \Theta(-x)-x-\lambda_{\mu}\right] \chi_{\mu}(x)=0 .
$$

Here $x=\xi / l_{0}, \lambda_{\mu}=\varepsilon_{\mu} / \varepsilon_{0}$, and $u_{0}=U_{0} / \varepsilon_{0}$. $\operatorname{Ai}(\xi)$ :

The regular solution of (61) is given by the Airy function

$$
\chi_{\mu}(x) \sim \begin{cases}\operatorname{Ai}\left(-x-\lambda_{\mu}\right) & \text { if } x>0 \\ \operatorname{Ai}\left(u_{0}-x-\lambda_{\mu}\right) & \text { if } x \leq 0\end{cases}
$$

Let us mention that the case of attractive cylinder potential $U_{0}$ could be practically realised if cold neutrons are scattered in the bulk of the media with positive optical potential $+U_{0}$ on a cylinder hole [4].

By matching the logarithmic derivatives of the "out" (incoming plus reflected wave, $\xi>0$ ) and "in" (wave function inside cylinder, $\xi<0$ ) solutions at $\xi=0$ one can get exactly the same equation for the $S$-matrix as (28) and, correspondingly, the same equation for the $S$-matrix poles as (34).

Thus the complex values of $x_{n}$ in (34) could be interpreted as the complex values of the radial energy $\lambda_{n}$ (in units $\varepsilon_{0}$ ) of the quasi-stationary centrifugal states of neutrons near the cylinder surface [17].

Let us mention that for extremely large angular momenta of interest neutron motion along angular variable $\varphi$ is classical; thus we can establish direct relation between angle and time: $\varphi \simeq \hbar \mu_{0} /\left(M R^{2}\right) t$. This turns the problem of neutron scattering on a cylinder into a problem of temporal evolution of resonant states in effective potential of the neutron radial motion.

For attractive potential there are states bound inside an effective potential well. These are quasi-stationary states given by (37). The quasi-stationary nature of such states is explained by the nonvanishing probability of the neutron penetration through the triangular barrier (Figure 3 ) into the mirror bulk. This probability strongly depends on the effective triangular barrier height $u_{0}$, which is a function of neutron velocity,

$$
u_{0}=\frac{U_{0}}{\varepsilon_{0}}=\frac{2 M U_{0}}{\hbar^{2} k^{2}}\left(\frac{\mu_{0}}{2}\right)^{2 / 3}
$$




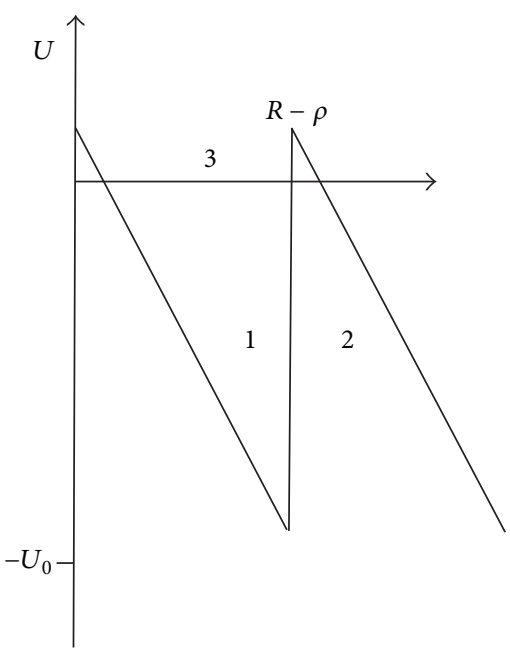

(a)

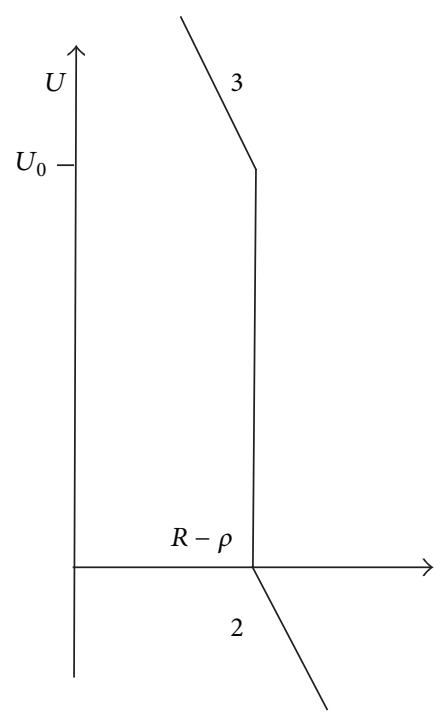

(b)

FIGURE 3: A sketch of the potential in the mirror surface vicinity is shown for the case of attractive (a) and repulsive (b) optical Fermipotentials. The potential step at $\xi=0$ is equal to the mirror optical potential $U_{0}$. The potential slope at $\xi \neq 0$ is governed by the centrifugal effective acceleration $a=v^{2} / R$. The regions, responsible for different type of resonances, are indicated with numbers: 1: long-living quasibound states, 2: surface waves, and 3: narrow over-barrier resonances.

and determines the width $\Gamma_{n}$ of the centrifugal state. These states are particularly important for a whispering gallery neutron scattering [4].

Another type of states, given by (42), is narrow overbarrier resonances. They are explained by phenomenon of socalled quantum reflection from the sharp edge of the effective potential. The condition of efficient quantum reflection consists in smallness of the characteristic scale of potential change compared to the wave-length of the neutron radial motion. It was shown in [4] that the widths of such resonances are particularly sensitive to the shape of the edge of effective potential, and its smoothing results in fast increase of the width of such states.

Let us mention that these narrow over-barrier resonances exist in case of both attractive and repulsive optical Fermipotential. In case of cold neutrons scattering on a cylinder of few centimeters radius they contribute to scattering to few degrees angles. Such states are analogous to the gravitational quasi-stationary states of antiatoms above material surface, predicted in $[18,19]$.

Finally, resonances given by (48) are responsible for phenomenon of so-called surface waves. They can be interpreted as a result of neutron evanescent wave reflection from the sharp edge of the optical Fermi-potential when neutron tunnels throw the centrifugal barrier and penetrate to the cylinder surface. Due to rapidly increasing width as a function of $n$ and correspondingly short lifetimes only few lowest states contribute to the neutron scattering at large angles.

Figure 3 illustrates the regions of effective potential, responsible for "production" of resonances of certain type.

\section{Conclusion}

We studied the position of Regge poles in complex momentum plane for the case of cold neutron scattering on a macroscopical curved mirror. Typical angular momenta which contribute to large angle scattering are of order of $10^{8}$, so asymptotic methods are required. We establish the corresponding equations for the Regge poles and find two types of such poles: narrow resonances and wide surface waves resonances. We establish the direct relation between the values of complex angular momenta, corresponding to Regge poles and complex radial energies of quasi-stationary states of neutron, bound near the curved surface by effective potential. The Regge poles imaginary part gives the width of such states. These states are particularly important for large angle scattering. Such scattering, determined by nearsurface neutron states, is particularly sensitive to the details of neutron-surface interaction. It could be a promising tool for studying such kind of interactions.

\section{Conflict of Interests}

The authors declare that there is no conflict of interests regarding the publication of this paper.

\section{References}

[1] V. V. Nesvizhevsky, A. Y. Voronin, R. Cubitt, and K. V. Protasov, "Neutron whispering gallery," Nature Physics, vol. 6, no. 2, pp. 114-117, 2010.

[2] V. V. Nesvizhevsky, A. K. Petukhov, K. V. Protasov, and A. Y. Voronin, "Centrifugal quantum states of neutrons," Physical 
Review A-Atomic, Molecular, and Optical Physics, vol. 78, no. 3, Article ID 033616, 2008.

[3] R. Cubitt, V. V. Nesvizhevsky, A. K. Petukhov et al., "Methods of observation of the centrifugal quantum states of neutrons," Nuclear Instruments and Methods in Physics Research A, vol. 611, no. 2-3, pp. 322-325, 2009.

[4] V. V. Nesvizhevsky, R. Cubitt, K. V. Protasov, and A. Y. Voronin, "The whispering gallery effect in neutron scattering," New Journal of Physics, vol. 12, Article ID 113050, 2010.

[5] J. W. S. Rayleigh, The Theory of Sound, vol. 2, Macmillan, London, UK, 1878.

[6] L. Rayleigh, "IX. Further applications of Bessel's functions of high order to the Whispering Gallery and allied problems," Philosophical Magazine, vol. 27, no. 157, p. 100, 1914.

[7] G. Mic, Annalen der Physik, vol. 25, p. 371, 1908.

[8] P. Debye, Annalen der Physik, vol. 30, p. 57, 1909.

[9] A. N. Oraevsky, "Whispering-gallery waves," Quantum Electronics, vol. 32, no. 5, pp. 377-400, 2002.

[10] K. J. Vahala, “Optical microcavities," Nature, vol. 424, no. 6950, pp. 839-846, 2003.

[11] H. M. Nussenzweig, Diffraction Effects in Semiclassical Scattering, Cambridge University Press, 1992.

[12] L. D. Landau and E. M. Lifshitz, Quantum Mechanics. Nonrelativistic Theory, Pergamon Press, London, UK, 1965.

[13] V. de Alfaro and T. Regge, Potential Scattering, North-Holland, Amsterdam, The Netherlands, 1965.

[14] H. M. Nussenzveig, Causality and Dispersion Relations, Academic Press, 1972.

[15] Y. Décanini and A. Folacci, "Resonant magnetic vortices," Physical Review A, vol. 67, Article ID 042704, 2003.

[16] M. Abramowitz and I. E. Stegun, Handbook of Mathematical Functions, Dover Publications, New York, NY, USA, 1965.

[17] A. I. Baz, Y. B. Zeldovich, and A. M. Perelomov, Scattering, Reactions and Decays in the Nonrelativistic Quantum Mechanics, Israel Program for Scientific Translations, Jerusalem, Israel, 1969.

[18] A. Y. Voronin, P. Froelich, and B. Zygelman, "Interaction of ultracold antihydrogen with a conducting wall," Physical Review A, vol. 72, no. 6, Article ID 062903, 2005.

[19] A. Y. Voronin, P. Froelich, and V. V. Nesvizhevsky, "Gravitational quantum states of Antihydrogen," Physical Review AAtomic, Molecular, and Optical Physics, vol. 83, no. 3, Article ID 032903, 2011. 

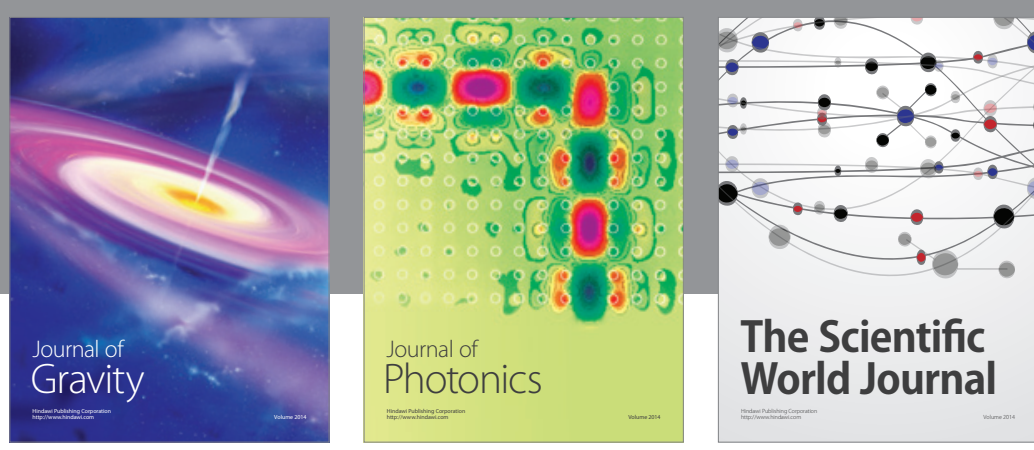

The Scientific World Journal
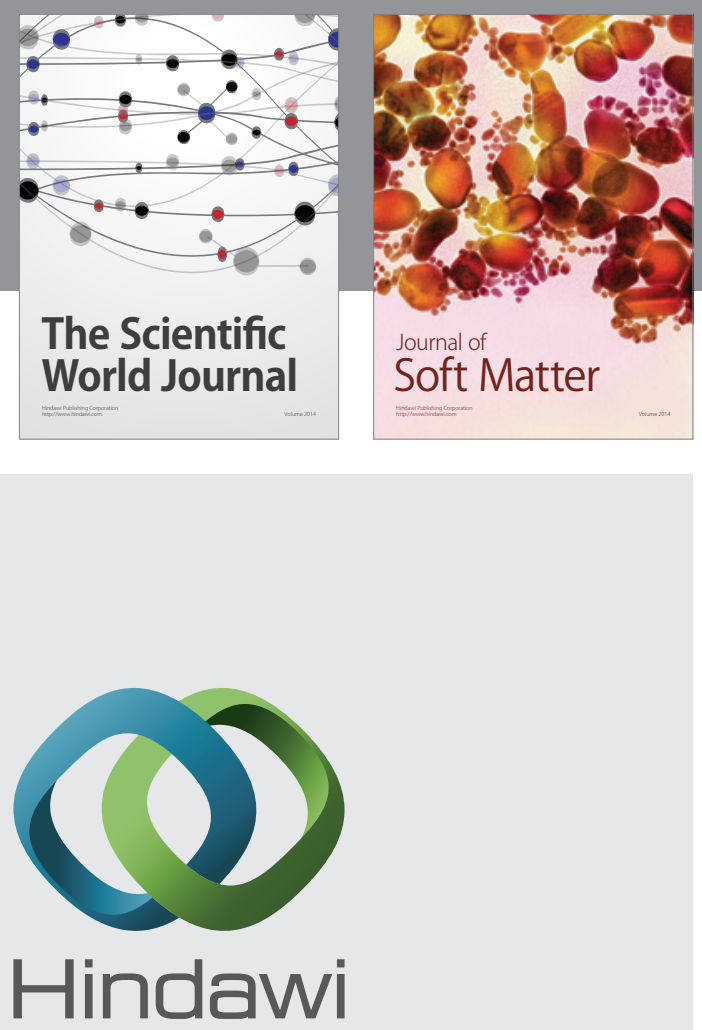

Submit your manuscripts at

http://www.hindawi.com

nternational Journal of

Statistical Mechanics
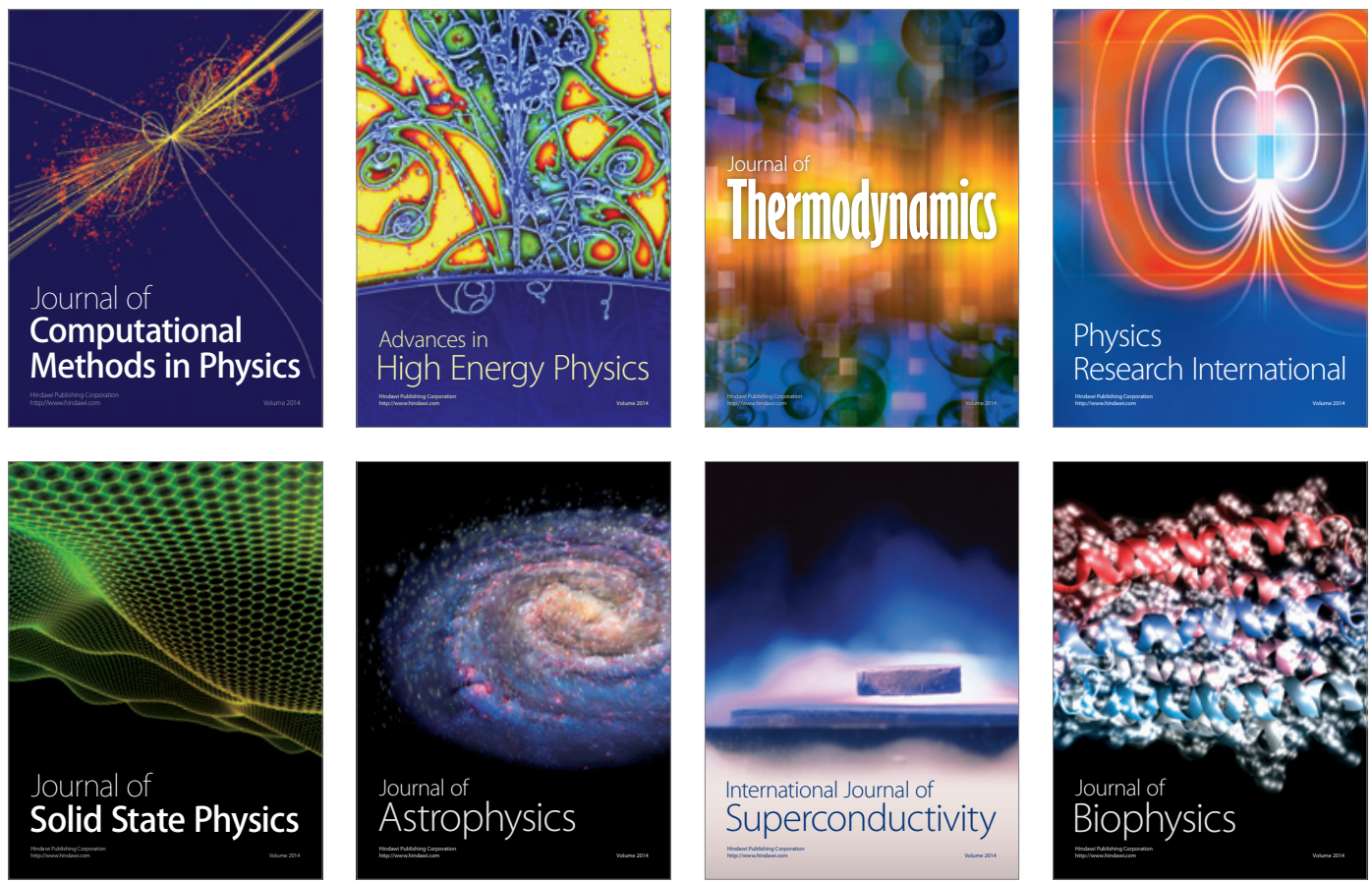
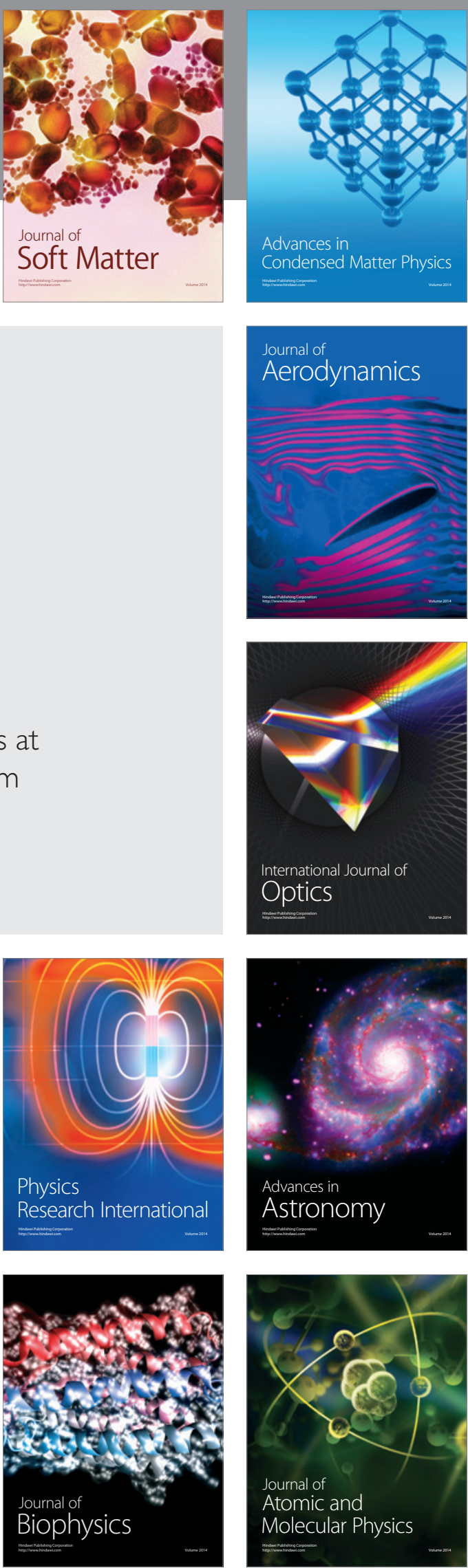